\title{
RF/THERMAL/STRUCTURAL ANALYSIS OF THE APT LEDA HOT MODEL CAVITY ASSEMBLY *
}

\author{
T. Schultheiss, M. Cole, J. Rathke, Advanced Energy Systems, Inc., Medford, NY \\ R. Wood, F. Krawczyk, P. Leslie, D. Schrage, Los Alamos National Laboratory, Los Alamos NM
}

\begin{abstract}
A three-dimensional ANSYS RF, Thermal, and Structural analysis has been performed for representative LEDA CCDTL RF structures. Geometry was taken from a Parasolid model that was generated by Los Alamos National Laboratory and represented one cavity structure. SUPERFISH results of this cavity were also provided. The ANSYS RF results were compared to SUPERFISH and to MAFIA models of the same cavity. Enough detail was used in the model to obtain heat load distributions for the coupling slot, coupling cavities, and accelerating cavities. These heat load distributions were then used as input for subsequent thermal analysis. Thermal analysis included coolant elements with heat transport capabilities and temperature dependent properties. Steady state temperature and stress distributions were determined at full field levels. Transient thermal analysis was completed to determine maximum thermal gradients and temperature distributions due to RF cycling. Structural analysis was completed to determine the locations of the model that were beyond yield strength. Plastic stress analysis from a single RF cycle was completed to determine permanent yield of the cavity.
\end{abstract}

\section{INTRODUCTION}

Three-dimensional modeling of the LEDA CCDTL cavities included determination of the heat load distribution from the RF analysis and applying these heat loads to subsequent thermal models.

Geometry for the analysis was taken from a Parasolid model provided by Los Alamos National Laboratory, figure 1 . The geometry represents cavity number 23 for which a SUPERFISH output was also provided.

The scaling method used for the ANSYS results was the same method that was used with SUPERFISH. The scale factor was determined by the ratio $\mathrm{E}_{0}$ normalization, (a given value) to the mean axial electric field, $\mathrm{E}_{0}$ defined as:

$$
E_{0}=\frac{1}{L} \int_{L / 2}^{L / 2} E(0, z) d z
$$

where $\mathrm{L}$ is the physics length of the CCDTL cell (4.1638 $\mathrm{cm})$ and $\mathrm{E}(0, \mathrm{z})$ is taken along the beam axis. The electric field profile after scaling from the 3-D ANSYS analysis

\footnotetext{
*Work sponsored by the University of California through the Los Alamos National Laboratory under Burns \& Roe Contract Number 2031-NMC-0031.
}

was compared to the SUPERFISH field profile. The magnetic fields resulting after scaling were used to determine the heat flux at the cavity surface.

Some interesting features of the RF model results include a frequency of $700.45 \mathrm{MHz}$, compared to the target structure frequency of $\sim 700 \mathrm{MHz}$. The model also shows a very high heat flux in the corner of the slot, heat flux increases on the cavity wall near the stem interface and small but possibly significant heat loads within the nominally unexcited coupling cavity.

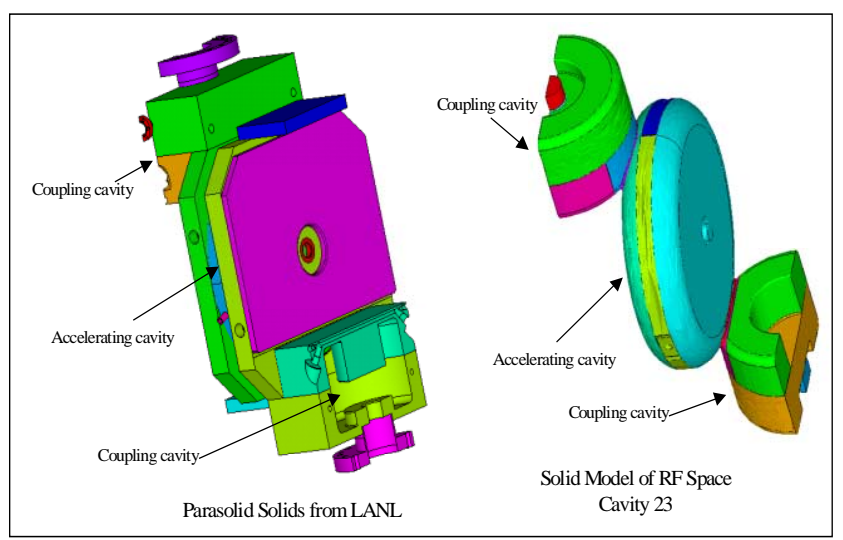

Figure 1, Solid model of structure to rf model space

\section{RF MODELING}

As much as possible, symmetry conditions were used to minimize the size of the model. The two coupling cavities include $1 / 4$ of each of their geometry and the accelerating cavity includes $1 / 2$ of its geometry. The magnetic field was assumed to be perpendicular to the cut planes of the cavities while the cavity surfaces were assumed to be perfect electric conductors. When solving for the frequencies and using a normal magnetic field condition for all the cut boundaries as discussed above, the mode representing a normal CCDTL $\pi / 2$ mode is suppressed. When the electric field tangent to the coupling cavity cut surface (electric normal, figure 2 ) is set to zero, the resulting mode represents the normal CCDTL $\pi / 2$ mode fields. The electric normal, electric wall, or perfect electric conductor boundary condition refers to setting the electric field components tangent to the surface to zero. This boundary condition also sets the magnetic field perpendicular to its surface to zero. The only allowed electric field is perpendicular to the surface. Due to the geometry of the coupling cavity the E field falls to zero at this surface, satisfying the $\pi / 2$ mode fields. 


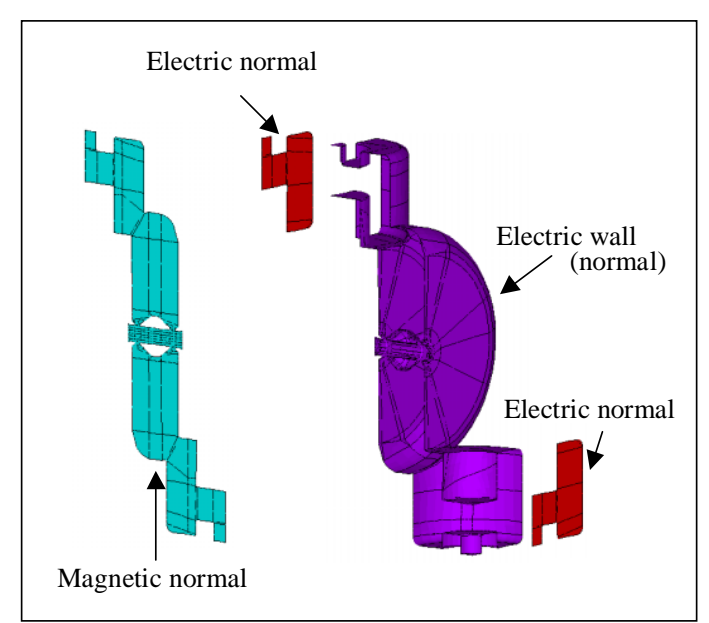

Figure 2, RF Boundary Conditions - $\pi / 2$ mode

The scaled electric field profiles of ANSYS and SUPERFISH are compared in Figure 3. The shape of the electric field is in excellent agreement which indicates an accurate scaling parameter for the ANSYS results. The $\mathrm{RF}$ analysis results were compared to SUPERFISH and MAFIA results generally with good agreement, Table 1 . Only in the drift tube noses where the electric field gradients were high do the results differ from SUPERFISH by more than $10 \%$. Away from the drift tube nose in the high magnetic field region the field results are in good agreement, signifying accurate heat loads.

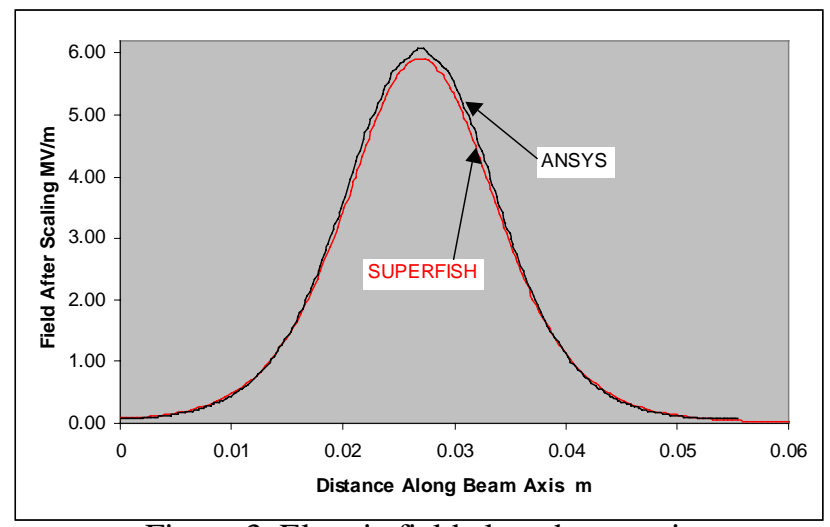

Figure 3, Electric field along beam axis

Table 1, RF results comparison

\begin{tabular}{|c|c|c|c|}
\hline & MAFIA & SUPERFISH & ANSYS \\
\hline $\mathrm{E}_{\mathrm{z}} \max \mathrm{MV} / \mathrm{m}$ & 6.5 & 5.9 & 6.09 \\
$\mathrm{U}_{\text {cav }} \mathrm{J}$ & .051 & .050 & .051 \\
$\mathrm{P}_{\text {cav }} \mathrm{kW}$ & 11.193 & 12.636 & 13.682 \\
$P_{\text {cc }} \max \mathrm{W} / \mathrm{cm}^{2}$ & .472 & N/A & .55 \\
$P_{\text {slot }} \max \mathrm{W} / \mathrm{cm}^{2}$ & 64.9 & N/A & 60.04 \\
\hline
\end{tabular}

The surface heat flux was determined from the magnetic field results and applied to the thermal model. Figure 4 shows a contour map of the heat loads which peak at the corners of the coupling cavity and the accelerating cavity. These corner loads were calculated to be 60.5 watts $/ \mathrm{cm}^{2}$. The next step in the analysis was to apply these loads to a thermal model to determine the temperatures and temperature gradients.

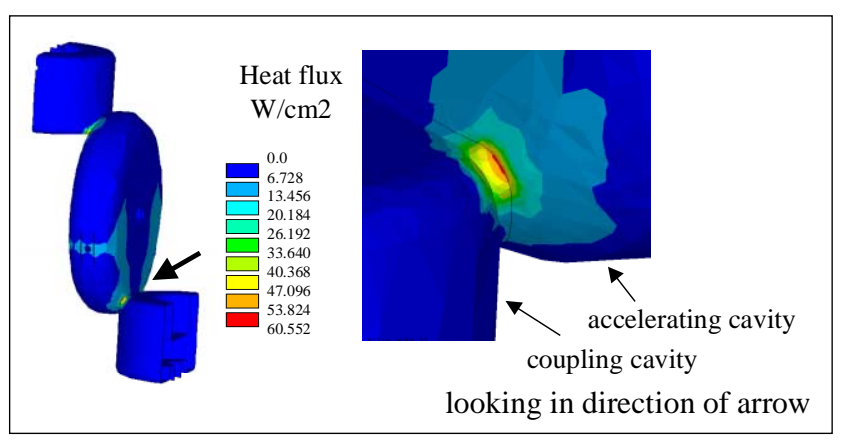

Figure 4, Heat flux on rf surfaces

\section{THERMAL ANALYSIS}

The structure, as shown in figure 1, was modeled with the element surfaces of the RF model matching the element surfaces of the thermal model. Therefore, the transfer of surface heat loads from the RF model can be made directly to the thermal model. If the surface elements didn't match, the heat loads could be mapped to the thermal model. Details of the thermal model include the coolant channels and one-dimensional fluid elements which account for fluid heat up and allow for flow balancing due to pressure drop.

Steady state temperature results are shown in figure 5. The temperatures shown in this figure are in Fahrenheit and indicate the effects of the increased heat loads in the slot between the accelerating and coupling cavities. Though these temperatures are not excessively high the local thermal gradients are large for a copper structure. At times gradients can be intensified due to a start up or a

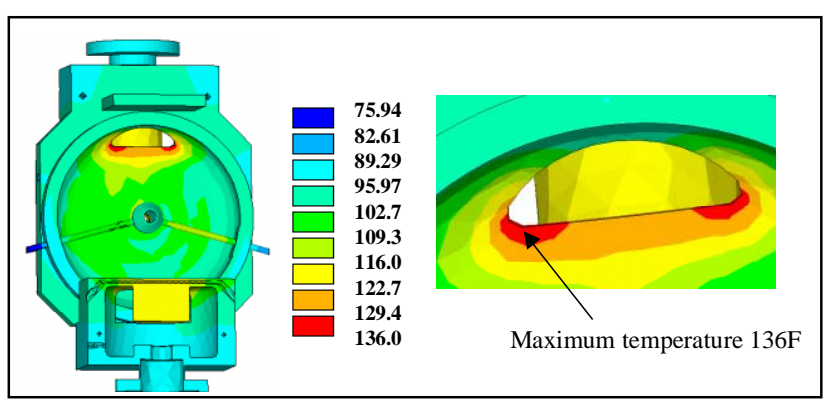

Figure 5, Temperature contours near upper slot

shut down power condition. Therefore, the thermal transient analyses were completed for both start up to steady state and shut down from steady state. Two different types of shut downs were examined. First the power was shut off abruptly with the coolant left on; second both the coolant and power were shut off abruptly. The model was run for a few hundred seconds until temperature differences between the slot and nearby 
locations became small. Temperature results are shown on figure 6 for the shut down response with the coolant left flowing. For this configuration the results showed that the gradients were largest at steady state. The results however, did show that temperature differences between the drift tube stem and the cavity were largest during shut down due to the stem responding at a much faster rate than the main structure, however, the resulting stresses were below yield strength.

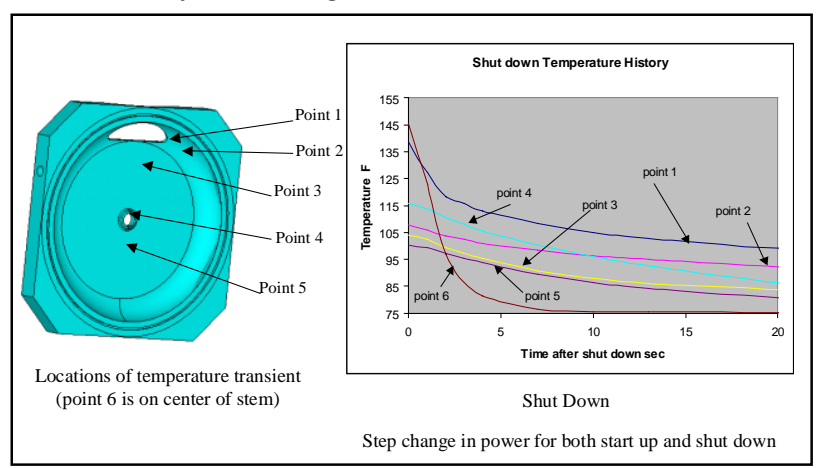

Figure 6, Shut down temperature response

\section{STRUCTURAL ANALYSIS}

The temperature distributions at numerous time points immediately following RF shutdown were used for input to the structural model. Stresses were then determined for these time points to find the time of maximum stress and to determine if locations other than the slot corners developed stresses above yield. All structural calculations assumed elastic properties. Stress histories were determined for the slot, coupling cavity, and stem. Results show that the maximum stress occurs in the coupling slot at steady state. The von Mises stress in the coupling slot corners is shown on figure 7 for steady state temperatures. These stresses are well above the design stress of $3500 \mathrm{psi}$ and are also above the yield strength of $7000 \mathrm{psi}$. These results indicate that plastic deformation will occur in the slot corners.

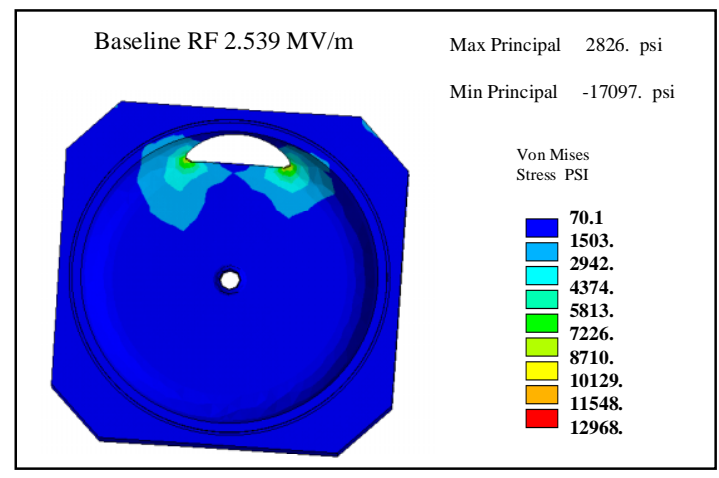

Figure 7, von Mises stress

\section{PLASTICITY ANALYSIS}

A single cycle RF power on to steady state and abrupt RF power off with the coolant flowing was analyzed to determine the residual plastic deformations within the coupling cavity slots. The nodal temperature histories were input at varying time points resulting in a smooth increase and decrease of the thermal loads. Ambient pressure was applied to the outside of the cavity throughout the analysis. To determine the residual deformation the pressure load is removed on the last plastic load step. Residual plastic deformation results are given in figure 8.

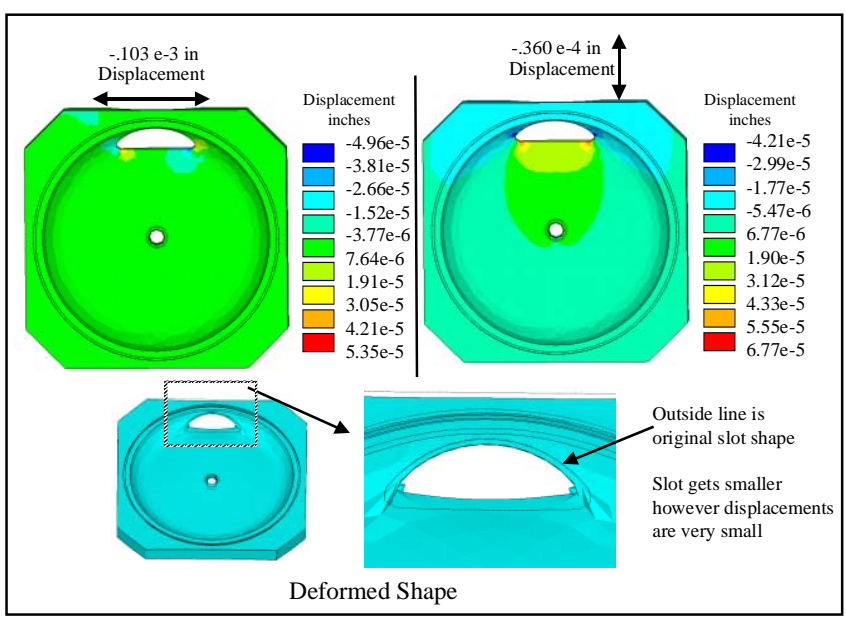

Figure 8, Permanent deformations

\section{CONCLUSIONS}

A RF/Thermal/Structural/Plastic analysis using a single set of nodes and elements was completed and residual plastic deformations were determined for a CCDTL structure. The analysis showed that the CCDTL though incurring plastic stress would result in small permanent deformations.

\section{REFERENCES}

[1] T. Schultheiss, M. Cole "RF Power Distribution Within the LEDA CCDTL Cavities When Operating in the $\pi / 2$ mode at full design fields"; AES, Inc. Report, Doc. No. AES-R-00-001, 1/10/00.

[2] T. Schultheiss, "Analysis to Determine Steady State Temperature and Stress Distributions of the APT LEDA Hot Model Cavity Assembly Operating at Full Field Levels"; AES, Inc. Report, Doc. No. AES-R$00-016,2 / 28 / 00$.

[3] T. Schultheiss, J. Rathke, "Analysis of the APT LEDA Hot Model Cavity Assembly With a Perturbation and Plasticity"; AES, Inc. Report, Doc. No. AES-R-00-073, 22/22/00.

[4] F. Krawczyk, "RF Power Levels in CCDTL Coupling Cavity," LANL memorandum LANSCE1:TN-00-007 2/7/00. 